\title{
Gait classification of patients with Fabry's disease based on normalized gait features obtained using multiple regression models
}

\author{
$1^{\text {st }}$ Carlos Fernandes \\ Dept. of Industrial Electronics, \\ University of Minho \\ Guimarães, Portugal \\ carlos.rafael.fernandes@hotmail.com
}

\author{
$3^{\text {th }}$ Miguel Gago \\ ICVS, School of Medicine \\ University of Minho \\ Braga, Portugal \\ miguelfgago@yahoo.com
}

\author{
$4^{\text {th }}$ Olga Azevedo \\ Cardiology Service \\ Hospital Senhora da Oliveira \\ Guimarães, Portugal
}

\author{
$5^{\text {th }}$ Nuno Sousa \\ ICVS, School of Medicine \\ University of Minho \\ Braga, Portugal \\ njcsousa@med.uminho.pt
}

\author{
$6^{\text {th }}$ Wolfram Erlhagen \\ Center of Mathematics \\ University of Minho \\ Guimarães, Portugal \\ wolfram.erlhagen@math.uminho.pt
}

\author{
$7^{\text {th }}$ Estela Bicho \\ Algoritmi Center, Dept. of Industrial Electronics, \\ University of Minho \\ Guimarães, Portugal \\ estela.bicho@dei.uminho.pt
}

\begin{abstract}
Diagnosis of Fabry disease (FD) remains a challenge mostly due to its rare occurrence and phenotipical variability, with considerable delay between onset and clinical diagnosis. It is then of extreme importance to explore biomarkers capable of assisting the earlier diagnosis of FD. There is growing evidence supporting the use of gait assessment in the diagnosis and management of several neurological diseases. In fact, gait abnormalities have previously been observed in FD, justifying further investigation. The aim of this study is to evaluate the effectiveness of different machine learning strategies when distinguishing patients with FD from healthy controls based on normalized gait features. Gait features of an individual are affected by physical characteristics including age, height, weight, and gender, as well as walking speed or stride length. Therefore, in order to reduce bias due to inter-subject variations a multiple regression (MR) normalization approach for gait data was performed. Four different machine learning strategies Support Vector Machines (SVM), Random Forest (RF), Multiple Layer Perceptrons (MLPs), and Deep Belief Networks (DBNs) - were employed on raw and normalized gait data. Wearable sensors positioned on both feet were used to acquire the gait data from 36 patients with FD and 34 healthy subjects. Gait normalization using MR revealed significant differences in percentage of stance phase spent in foot flat and pushing $(p<$ 0.05), with FD presenting lower percentages in foot flat and higher in pushing. No significant differences were observed before gait normalization. Support Vector Machine was the superior classifier achieving an FD classification accuracy of $78.21 \%$ after gait normalization, compared to $71.96 \%$ using raw gait data. Gait normalization improved the performance of all classifiers. To the best of our knowledge, this is the first study on gait classification that includes patients with FD, and our results support the use of gait assessment on the clinical assessment of FD.
\end{abstract}

Index Terms-Multiple regression models; Machine learning; Walking; Fabry's disease.

This work was partially supported by the projects NORTE-01-0145FEDER- 000026 (DeM-Deus Ex Machina) financed by NORTE2020 and FEDER, and the Pluriannual Funding Programs of the research centres CMAT and Algoritmi.

\section{INTRODUCTION}

Fabry Disease (FD) is a rare and progressive disease that greatly affects the quality of life and may lead to premature death. This disease is an X-linked lysosomal storage disorder caused by the deficiency or absent activity of the enzyme $\alpha$-Galactosidase A ( $\alpha$-Gal A). Progressive accumulation of neutral glycosphingolipids, primarily globotriaosylceramide (Gb3), in cells throughout the body leads to multisystem pathology [16], [22]. The most severe clinical manifestations of FD associated with life-threatening complications are the damages to the kidneys, heart, and brain. Cerebrovascular complications caused by cerebral vasculopathy are a major cause of morbidity and premature death in patients with FD [22]. The diagnosis of FD remains a challenge due to rare occurrence, symptom variability, different ages of onset, and severity of progression. The average delay between onset and correct diagnosis of FD is 13.7 and 16.3 years and premature death occurs on average 20 and 15 years earlier, in male and female patients, respectively [16]. It is then of extreme importance to explore different biomarkers that can assist in the early diagnose of FD. Although the involvement of motor function is not included in the manifestations typically associated with FD, motor impairments during gait, such as postural instability and slower gait, have been demonstrated in FD [25]. Furthermore, the most common FD manifestations are neurologic and gait has been hypothesized as the final outcome of several neurological functions [5].

There has been growing evidence showing that gait assessment can be a powerful complementary tool in the diagnosis and management of patients with motor impairments [13], [23], [26]. Gait features can be measured easily, quickly, and at low cost using noninvasive technology such as wearable 
sensors. Different machine learning methods based on gait features have been widely used to discriminate between normal and abnormal gait patterns and among different pathological gait patterns such as presented in patients with Parkinson's disease, Huntington's disease, and Alzheimer's disease. Some examples of recently used machine learning methods for gait classification include Support Vector Machines (SVM) [3], [27], [30], [32], [34], [35], Random Forest (RF) [3], [30], [34], [35], and Artificial Neural Networks (ANNs) [13], [32], [35]. Good classification performance has been found in the classification of pathological gait. To the best of our knowledge, no studies have been conducted that have tried to apply these algorithms to patients with FD.

Gait characteristics of a subject are affected by his physical properties including age, gender, height, and weight, as well as by walking speed [33], [34]. In [33], [34] and more recently in [28], a multiple regression (MR) normalization method was employed on gait data to minimize the effect of inter-subject physical differences and self-selected speed. Comparing to other methods, such as dimensionless equations and detrending methods, MR normalization revealed better results on reducing the interference of subject-specific physical properties and its gait variables, thereby improving parkinsonian gait classification accuracy using machine learning methods [34]. It has been shown that the accuracy of Parkinson's disease diagnosis using SVM and RF approaches improves from $81 \%$ to $89 \%$ and $75 \%$ to $93 \%$, respectively, when gait data is normalized using the MR approach [34]. In this study, we implement Wahid et al.'s method [33] to normalized various gait features. As in [28], [33], [34] age, height, weight, gender, and selfselected walking speed were used as independent variables. Additionally, we also included the subjects' stride length as an independent variable, as it was shown to significantly affect foot clearance gait features [4], [14].

The aim of the current study is twofold, firstly, use MR normalization approach to identify differences in gait features between FD patients and healthy subjects, and secondly, to evaluate the effectiveness of different machine learning methods in classifying FD gait after applying MR normalization. We hypothesized that it will be possible to distinguish FD gait from healthy controls using machine learning approaches and that by applying MR normalization to gait features classification performance will be improved. The results of this study have future implications for the use of the gait assessment as a complementary non-invasive tool for FD diagnosis and evaluation.

\section{Materials And Methods}

\section{A. Experimental Protocol}

Data from thirty-six FD patients ( 24 females and 12 males) and thirty-four healthy subjects (21 females and 13 males) were collected. For all FD patients, the exclusion criteria were: the presence of resting tremor, moderate-severe dementia $(\mathrm{CDR}>2)$, depression, less than eighteen years of age, extensive intracranial lesions or neurodegenerative disorders, musculoskeletal disease and rheumatological disorders. Local hospital ethics committee approved the protocol of the study, submitted by ICVS/UM and Center Algoritmi/UM. Written consent was obtained from all subjects or their guardians.

Two Physilog ${ }^{\circledR}$ sensors (Gait Up ${ }^{\circledR}$, Switzerland) positioned on both feet were used to measure different gait variables of each stride (also known as gait cycle). The participants were asked to walk 60-meter continuous course (30 meters corridor with one turn) in a self-selected walking speed while the sensors were doing the data acquisition. The average walking speed of FD patients and controls was $1.33 \pm 0.18$ $\mathrm{m} / \mathrm{s}$ and $1.33 \pm 0.15 \mathrm{~m} / \mathrm{s}$, respectively. The information about the participant age, weight, height, and body mass index were also collected. These physical characteristics are summarized in Table I.

TABLE I

AGE AND ANTHROPOMETRIC CHARACTERISTICS

\begin{tabular}{l|c|c|} 
& Controls & FD Patients \\
\hline Age $($ years $)$ & $53.32 \pm 23.45$ & $49.42 \pm 18.00$ \\
Weight $(\mathrm{kg})$ & $68.39 \pm 9.42$ & $65.67 \pm 9.17$ \\
Height $(\mathrm{m})$ & $1.68 \pm 0.092$ & $1.62 \pm 0.084$ \\
BMI $\left(\mathrm{kg} / \mathrm{m}^{2}\right)$ & $24.39 \pm 2.92$ & $25.16 \pm 3.63$ \\
\hline
\end{tabular}

Characteristics are displayed as mean \pm standard deviation. BMI - Body mass index.

\section{B. Data analysis}

The arithmetic mean of each gait variable was calculated for all subjects' stride time series. The collected gait variables used in this work are: speed (velocity of one cycle), cycle duration (duration of one gait cycle), cadence (number of gait cycles in a minute), stride length (distance between successive initial ground contact of the same foot), stance (percentage of the gait cycle where the foot is on the ground), swing (percentage of the gait cycle where the foot is in the air), loading (percentage of stance between the heel strike and the foot being fully on the ground), foot flat (percentage of stance where the foot is fully at on the ground), pushing (percentage of stance between the foot being fully on the ground and the toe leaving the ground), double support (percentage of the gait cycle where both feet touch the ground), peak swing (maximum angular velocity during swing), strike angle (angle between the foot and the ground when the heel hits the ground), lift-off angle (angle between the foot and the ground when the toes are leaving the ground), maximum heel clearance (maximum height above the ground reached by the heel), maximum toe clearance 1 (maximum height above the ground reached by the toes after heel max clearance), minimum toe clearance (minimum height of the toes during swing phase) and maximum toe clearance 2 (maximum height above the ground reached by the toes just before heel strike).

Variance inflation factors (VIF) for the independent gait variables were calculated to determine the severity of multicollinearity among the physical properties, speed and stride length. The VIFs for all combinations of independent variables are summarized in Table II. 
TABLE II

VARIANCE INFLATION FACTORS FOR AGE, HEIGHT, WEIGHT, GENDER, SPEED AND STRIDE LENGTH.

\begin{tabular}{ccccccc}
\hline & Age & Height & Weight & Gender & Speed & Stride Length \\
\hline \multirow{4}{*}{ VIF } & 2.45 & 3.85 & 1.94 & 1.27 & 4.13 & 8.21 \\
& 1.89 & 2.88 & 1.92 & 1.26 & 1.26 & - \\
& 2.24 & 3.45 & 1.91 & 1.26 & - & 2.51 \\
\hline
\end{tabular}

A VIF value is greater than 5 (8.21 for stride length) when considering all six independent variables. Then, speed and stride length independent variables are never considered simultaneously. Physical characteristics combined with speed or stride length were considered in the development of the MR models since their VIFs were less than 5 [7]. For each gait variable, the best regression model was selected based on adjusted $R^{2}$ and Akaike information criterion (AIC) values.

The Spearman's rank-order correlation coefficients $(\rho)$ were computed to assess the influence of the independent variables on the spatial-temporal and foot clearance gait variables before and after MR normalization. The correlations between the gait variables and gender were computed using a point biserial coefficient of correlation method [24].

\section{Data Normalization}

Spatial-temporal gait variables including gait cycle duration, cadence, stance, swing, loading, foot flat, pushing, double support, stride length, peak swing, and foot clearance gait variables including strike angle, lift-off angle, maximum heel clearance, maximum toe clearance 1 , minimum toe clearance and maximum toe clearance 2 were normalized to subject physical properties as follows:

$$
\hat{y}_{i}=\beta_{0}+\sum_{j=1}^{p} \beta_{j} x_{i j}+\varepsilon_{i}
$$

where $\hat{y}_{i}$ represents the prediction for the dependent gait variable for the $i$ th observation; $x_{i j}$ represents the $j$ th physical property including age, height, weight, gender, and speed or stride length, $\beta_{0}$ represents the intercept term, $\beta_{j}$ represents the coefficient for the $j$ th physical property and $\varepsilon_{i}$ represents the residual error for the $i$ th observation.

The coefficients of the MR model are estimated using the control subjects dataset. The best fitted MR models are then used to normalize each gait variable by dividing the value of the original dependent gait variable, $y_{i}$, by the predicted gait variable from (1), $\hat{y}_{i}$, according to:

$$
y_{i}^{n}=\frac{y_{i}}{\hat{y}_{i}}
$$

where $y_{i}^{n}$ represents the normalized gait variable for the $i$ th observation.

\section{Feature extraction}

Before feature extraction all gait variables were scaled to have zero mean and unit variance. Principal component analysis (PCA) was applied to both raw and MR normalized
16 gait variables for feature extraction. PCA is a method that projects the original set of features into the directions of the highest variance [2], [31]. This method extracts meaningful information from the original set of features and expresses it as a new set of orthogonal features called principal components (PCs). The PCs are linear combinations of the initial data features and are sorted in decreasing order of significance. The first principal component explains the maximum variance and each principal component that follows it explains a lower variance. A percentage of the total variance is set as threshold to select the number of principal components. PCA is one of the most fundamental and successful methods for dimensionality reduction. Using PCA to reduce data dimensionality results in a reduction of computational cost and may increase the performance of the machine learning classifiers [2], [17]. For each dataset, the principal components were selected based on a threshold of around $95 \%$ of the total explained variance.

\section{E. Machine Learning}

In this work, four classifier architectures were employed for the differentiation task of controls vs. FD patients' gait patterns. The implemented classifiers were Support Vector Machines (SVMs) [8], Random Forests (RFs) [9], Multilayer Perceptrons (MLPs) [19] and Deep Belief Networks (DBNs) [15], [20]. It is impossible to predict a priori which classifier will obtain the best classification performance for a given problem, so the implementation of different types of classifiers is recommended [17]. The machine learning classifiers were implemented using: 1) raw spatial-temporal and foot clearance gait variables; 2) MR normalized spatial-temporal and foot clearance gait variables.

The hyperparameters of each classifier were optimized using a randomized search method and 10-fold cross-validation. For the SVM classifiers, different configurations between the regularization $C$, Kernel functions and Kernel functions specific hyperparameters (e.g. degree of the polynomial kernel function) were evaluated. For the RF classifiers different configurations between the number of trees, the functions to measure the quality of a split (Gini or Entropy), the minimum number of samples required to split an internal node, the minimum number of samples required to be a leaf node and the maximum number of features to consider at each node split were evaluated. Finally, for the DBN and MLP classifiers different configurations between the number of hidden neurons, learning rates, dropout rates and epochs were evaluated. Both MLP and DBN classifiers were implemented with two hidden layers. The TensorFlow [1], Keras [12] and scikit-learn [29] libraries within the Python programming language were used to develop and implement the classifiers.

To evaluate the performance of the different classifiers a set of metrics including Accuracy (i.e., the proportion of the total number of classifications that were correctly classified), Specificity (i.e., the proportion of actual negative cases which are correctly classified), and Sensitivity (i.e., the proportion of actual positive cases which are correctly classified), and area under curve (AUC) were used. 
Sensitivity and Specificity are very useful in assessing the risks and benefits associated with a classifier. The cost associated with a false negative, in this case incorrectly predicting that an FD patient is a control subject, is far greater than the cost of a false positive. The receiver operating characteristic (ROC) curve is a tool used to analyze the trade-off between sensitivity and specificity [17]. Associated with the ROC curve is the area under the curve (AUC), this measure indicates the performance of a classifier using varying threshold values. The AUC value lies between 0.5 to 1 , where 1 denotes a perfect classifier and 0.5 represents the worst possible classification.

\section{RESULTS}

Normalization using the MR approach (see Table III) was able to reduce the great majority of correlations between the independent variables and gait variables, however few strong correlations remained after normalization (Table IV and Table V). For both groups, controls and FD, all raw gait variables were strongly correlated with at least one of the independent variables: age, weight, height, speed, gender, stride length and speed. Regarding the control subjects (Table IV), after normalization all gait variables were weakly correlated except the cycle duration which was mildly correlated with stride length $(\rho=0.39)$ and maximum toe clearance 1 which was mildly correlated with age $(\rho=-0.38)$. Concerning to the FD patients (Table V), after normalization the percentage of gait cycle in stance and swing were still strongly correlated with speed $(\rho=-0.42, \rho=0.43)$ and gender $(\rho=-0.48$, $\rho=0.48$ ), the percentage of gait cycle in double support was strongly correlated with speed $(\rho=-0.53)$, peak swing was strongly correlated with age $(\rho=-0.51)$, speed $(\rho=0.52)$ and stride length $(\rho=0.39)$, and finally strike angle was mildly correlated with weight $(\rho=0.41)$.

Using raw data, no statistically significant differences were found. After normalization using MR approach, significant differences between controls and FD patients were observed in foot flat (mean difference: $0.11,95 \% \mathrm{CI}$ : [0.10;0.14], $p=$ .011 ) and pushing (mean difference: 0.10, 95\%CI: [0.08,0.12], $p=.019$ ), with FD presenting lower percentages in foot flat and higher in pushing.

Classification accuracy of FD gait using machine learning was lowest when using the principal components (PCs) extracted from the raw gait variables, and highest when based on the PCs extracted from the MR normalized gait variables (see Table VI). The SVM classifier performed the best, it was able to yield an accuracy of $78.21 \%$ (76.89\% sensitivity and $88.50 \%$ specificity) in predicting FD gait based on the PCs extracted from the MR normalized gait. The MLP, DBN and RF classifiers based on the PCs extracted from MR normalized gait achieved an accuracy of $72.20 \%, 65.12 \%$ and $76.37 \%$, respectively.

ROC curves for all machine learning strategies are shown in Figure 2. The maximum AUC value (0.78) was yielded by the SVM classifier when based on the MR normalized gait PCs. The AUC values of all classifiers also increased when using the MR normalized gait PCs, the only exception was the AUC value for the DBN classifier which remained the same $(0.61)$ for both raw and MR normalized gait datasets.

Peak machine learning performances (both classification accuracies and AUC value) were observed when using the PCs extracted from gait data normalized by the MR approach.

\section{DISCUSSION}

The developed work was twofold, firstly it aimed to investigate the capabilities of MR normalization approach for de-correlation between spatial-temporal and foot clearance gait variables, physical properties, speed and stride length. Secondly, it evaluated the performance of machine learning classifiers based on raw and MR normalized gait variables when distinguishing FD patients and control subjects.

After gait data normalization using MR approach weak to moderate correlations $(0.00<|\rho|<0.49)$ were observed between physical characteristics, speed, stride length, and gait features, while for raw gait data these correlations were weak to strong $(0.01<|\rho|<0.82)$. Strong correlations between most of spatial-temporal gait variables and speed were observed, especially in FD patients. After MR normalization all correlations decreased to weak or moderate values $(0.00<|\rho|<0.54)$. Speed has shown a profound role in predicting spatial-temporal gait variables, all the MR models make use of speed for spatial-temporal prediction (see Table III). This goes in line with the hypothesis that normal ranges for gait variables should be defined with reference to speed of walking [21]. Speed has also been correlated with most spatial-temporal gait variables previously [6], [28], [33], [34]. Another study also concluded that differences in gait parameters between healthy subjects and osteoarthritis patients decrease when walking speed is accounted for in the gait analysis [36]. Additionally, in this work stride length was included as an independent variable for the development of the MR models. Stride length has been shown to affect gait variables, more specifically foot clearance measurements [4], [14]. Our results show that foot clearance measurements from FD patients and controls were strongly correlated with stride length, the variables most correlated with stride length were strike angle $(\rho=0.73$ and $\rho=0.66)$, lift-off angle $(\rho=-0.77$ and $\rho=-0.77)$ and maximum toe clearance $2(\rho=0.69$ and $\rho=0.77)$. After MR normalization, using stride length as an independent variable, the correlation between stride length, strike angle, lift-off angle and maximum toe clearance 2 were all weakened to non significant values $(|\rho|<0.25$ and $|\rho|<0.18)$. In fact, the regression models for strike angle, lift-off angle and maximum toe clearance 2 explain $54.2 \%$ to $66.0 \%$ in the observed variance using stride length as an independent variable (Table III). Stride length seems to be the strongest predictor for foot clearance variables, suggesting that future gait evaluation should account for the effect of stride length on those variables. In this work, the developed MR models that are best able to predict foot clearance patterns have stride length as an independent variable. Overall the MR normalization was able to significantly reduce the correlations between physical properties, speed, stride length and gait 
TABLE III

RESULTING MULTIPLE LINEAR REGRESSION MODELS FOR THE GAIT VARIABLES. THE ADJUSTED $R^{2}$ AND AKAIKE INFORMATION CRITERION (AIC) ARE

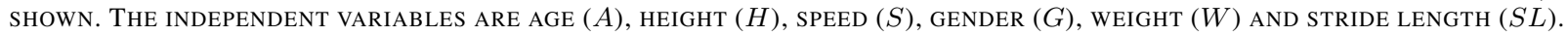

\begin{tabular}{|c|c|c|c|c|c|c|c|c|c|}
\hline Gait variable & \multicolumn{7}{|c|}{ Multiple Linear Regression Model } & AIC & Ajusted $R^{2}$ \\
\hline \multicolumn{10}{|c|}{ Spatial-Temporal Variables } \\
\hline Cycle Duration & $=1.04$ & $-0.00095 \cdot A$ & $+0.277 \cdot H$ & $-0.308 \cdot S$ & & & & -109.19 & 0.615 \\
\hline Cadence & $=112.22$ & $+0.130 \cdot A$ & $-29.0 \cdot H$ & $+34.96 \cdot S$ & & & & 203.12 & 0.680 \\
\hline Stance & $=62.44$ & & & $-3.35 \cdot S$ & $+1.41 \cdot G$ & & & 132.17 & 0.188 \\
\hline Swing & $=37.60$ & & & $-3.35 \cdot S$ & $-1.41 \cdot G$ & & & 132.17 & 0.188 \\
\hline Loading & $=22.37$ & & & $+3.32 \cdot S$ & $-2.30 \cdot G$ & $-0.154 \cdot W$ & & 154.73 & 0.315 \\
\hline Foot Flat & $=78.31$ & & $-8.95 \cdot H$ & $-16.59 \cdot S$ & & $+0.194 \cdot W$ & & 176.89 & 0.543 \\
\hline Pushing & $=17.23$ & $-0.036 \cdot A$ & & $+13.25 \cdot S$ & & & & 168.30 & 0.469 \\
\hline Double Support & $=25.57$ & & & $-6.41 \cdot S$ & $+1.81 \cdot G$ & & & 176.12 & 0.121 \\
\hline Stride Length & $=0.24$ & $-0.0018 \cdot A$ & $+0.361 \cdot H$ & $+0.455 \cdot S$ & & & & -105.97 & 0.864 \\
\hline Peak Swing & $=231.52$ & & $-41.22 \cdot H$ & $+129.90 \cdot S$ & & $+0.833 \cdot W$ & & 305.71 & 0.526 \\
\hline \multicolumn{10}{|c|}{ Foot Clearance Variables } \\
\hline Strike Angle & $=19.24$ & & & & $-3.03 \cdot G$ & $-0.165 \cdot W$ & $+16.51 \cdot S L$ & 167.54 & 0.542 \\
\hline Lift-Off Angle & $=-46.51$ & $+0.218 \cdot A$ & & & & & $-0.137 \cdot S L$ & 206.11 & 0.660 \\
\hline MaxHC & $=0.101$ & & $+0.17 \cdot H$ & & $-0.039 \cdot G$ & $-0.00091 \cdot W$ & & -137.25 & 0.474 \\
\hline MaxTC1 & $=0.136$ & & & & $-0.022 \cdot G$ & $-0.00055 \cdot W$ & & -143.17 & 0.078 \\
\hline MinTC & $=-0.015$ & $+0.00049 \cdot A$ & $+0.0121 \cdot H$ & & & & & -184.17 & 0.309 \\
\hline MaxTC2 & $=0.095$ & $-0.00070 \cdot A$ & & & $-0.033 \cdot G$ & & $+0.110 \cdot S L$ & -142.60 & 0.602 \\
\hline
\end{tabular}

MaxHC: Maximum Heel Clearance; MaxTC1: Maximum Toe Clearance 1; MinTC: Minimum Toe Clearance; MaxTC2: Maximum Toe Clearance 2.

TABLE IV

SPEARMAN CORRELATION COEFFICIENTS FOR THE CONTROLS GAIT DATA BEFORE (RAW) AND AFTER MR NORMALIZATION.

\begin{tabular}{|c|c|c|c|c|c|c|c|c|c|c|c|c|}
\hline \multirow{2}{*}{ Correlations } & \multicolumn{2}{|c|}{ Age } & \multicolumn{2}{|c|}{ Weight } & \multicolumn{2}{|c|}{ Height } & \multicolumn{2}{|c|}{ Speed } & \multicolumn{2}{|c|}{ Stride Length } & \multicolumn{2}{|c|}{ Gender } \\
\hline & Raw & Norm & Raw & Norm & Raw & Norm & Raw & Norm & Raw & Norm & Raw & Norm \\
\hline \multicolumn{13}{|l|}{ Spatial-Temporal Variables } \\
\hline Cycle Duration & -0.28 & -0.05 & 0.28 & 0.23 & 0.46 & 0.26 & -0.36 & -0.02 & 0.18 & 0.39 & -0.29 & -0.11 \\
\hline Cadence & 0.3 & 0.11 & -0.3 & -0.27 & -0.45 & -0.27 & 0.35 & 0.02 & -0.18 & -0.41 & 0.28 & 0.1 \\
\hline Stance & 0.13 & 0.0 & 0.01 & 0.14 & -0.08 & 0.18 & -0.27 & -0.01 & -0.29 & -0.01 & 0.37 & 0.0 \\
\hline Swing & 0.13 & -0.03 & -0.01 & -0.15 & 0.08 & -0.18 & 0.27 & -0.01 & 0.29 & 0.01 & -0.37 & -0.01 \\
\hline Loading & -0.05 & 0.04 & -0.38 & -0.0 & -0.09 & 0.01 & 0.12 & 0.0 & 0.08 & 0.03 & -0.26 & 0.01 \\
\hline Foot Flat & 0.42 & 0.1 & 0.34 & 0.04 & -0.16 & -0.11 & -0.64 & -0.25 & -0.58 & -0.29 & 0.03 & 0.17 \\
\hline Pushing & -0.44 & -0.01 & -0.06 & -0.12 & 0.3 & -0.05 & 0.68 & 0.14 & 0.62 & 0.11 & 0.15 & 0.23 \\
\hline Double Support & 0.07 & -0.04 & 0.12 & 0.2 & 0.0 & 0.15 & -0.23 & -0.01 & -0.23 & -0.03 & 0.25 & -0.01 \\
\hline Stride Length & -0.63 & -0.07 & 0.23 & 0.21 & 0.68 & 0.24 & 0.82 & 0.28 & 1.0 & 0.56 & -0.11 & -0.02 \\
\hline Peak Swing & -0.03 & 0.13 & 0.21 & 0.01 & 0.25 & 0.05 & 0.69 & 0.14 & 0.57 & 0.16 & -0.02 & -0.07 \\
\hline \multicolumn{13}{|l|}{ Foot Clearance Variables } \\
\hline Strike Angle & -0.44 & -0.05 & -0.1 & 0.06 & 0.36 & 0.07 & 0.49 & 0.06 & 0.66 & 0.18 & -0.33 & 0.01 \\
\hline Lift-Off Angl & 0.7 & -0.03 & -0.08 & -0.05 & -0.54 & 0.08 & -0.65 & 0.13 & -0.77 & 0.15 & 0.12 & -0.06 \\
\hline Maximum Heel Clear & -0.38 & -0.22 & 0.22 & 0.1 & 0.59 & 0.18 & 0.27 & 0.12 & 0.52 & 0.27 & -0.56 & -0.02 \\
\hline Maximum Toe Clearance 1 & 0.3 & 0.36 & -0.01 & 0.14 & -0.07 & -0.11 & -0.33 & -0.27 & -0.2 & -0.18 & -0.32 & 0.01 \\
\hline Minimum Toe Cleara & 0.66 & 0.07 & 0.02 & -0.05 & -0.34 & -0.17 & -0.42 & -0.23 & -0.47 & -0.17 & 0.09 & 0.03 \\
\hline Maximum Toe Clearance 2 & -0.62 & 0.02 & 0.08 & -0.19 & 0.59 & -0.08 & 0.54 & 0.05 & 0.77 & 0.11 & -0.45 & -0.02 \\
\hline
\end{tabular}

variables. The MR normalization results of this work are comparable to previous works and further corroborate the favorable outcomes of using MR normalization [28], [33], [34].

The interpretation of Figure 1 indicates that the MR normalization was able to uncover significant differences in foot flat and pushing which indicates that MR normalization would improve the classification performance of FD gait patterns. This hypothesis is corroborated by the performance of the developed classifiers. All machine learning classifiers performed better based on the MR normalized variables, SVM was the best classifier achieving an accuracy of $78.2 \%$, a sensitivity of $76.9 \%$ and a specificity of $88.5 \%$, the accuracy of the SVM classifier increased $6.3 \%$ when MR normalization is applied before classification (see Table VI). The SVM classifier displayed the most consistent and best performances among all classifiers both based on raw and normalized gait, these results are in accordance with previous studies that used gait variables to classify gait patterns of various neurological disorders. A previous study used various machine learning classifiers to differentiate between Parkinson's disease and Alzheimer's disease gait patterns, the best performance was achieved by the SVM classifier ( $92.6 \%$ accuracy) [3]. Another study compared the performance of SVM, MLP, RF, and k-NN when differentiating between controls and neurodegenerative disorders based on gait rhythm signals, the best classifier also was the SVM with an accuracy of $96.83 \%$ [35].

Our results also show that RF has an impressive per- 
TABLE V

SPEARMAN CORRELATION COEFFICIENTS FOR THE FD PATIENTS GAIT DATA BEFORE (RAW) AND AFTER MR NORMALIZATION.

\begin{tabular}{|c|c|c|c|c|c|c|c|c|c|c|c|c|}
\hline \multirow{2}{*}{ Correlations } & \multicolumn{2}{|c|}{ Age } & \multicolumn{2}{|c|}{ Weight } & \multicolumn{2}{|c|}{ Height } & \multicolumn{2}{|c|}{ Speed } & \multicolumn{2}{|c|}{ Stride Length } & \multicolumn{2}{|c|}{ Gender } \\
\hline & Raw & Norm & Raw & Norm & Raw & Norm & Raw & Norm & Raw & Norm & Raw & Norm \\
\hline \multicolumn{13}{|l|}{ Spatial-Temporal Variables } \\
\hline Cycle Duration & 0.12 & 0.06 & 0.38 & 0.25 & 0.32 & 0.24 & -0.63 & -0.24 & -0.08 & 0.27 & -0.46 & -0.37 \\
\hline Cadence & -0.16 & -0.17 & -0.43 & -0.28 & -0.4 & -0.34 & 0.63 & 0.24 & 0.08 & -0.26 & 0.43 & 0.34 \\
\hline Stance & 0.36 & 0.11 & 0.19 & 0.29 & -0.05 & 0.26 & -0.66 & -0.42 & -0.39 & -0.07 & -0.15 & -0.48 \\
\hline Swing & -0.36 & -0.13 & -0.19 & -0.29 & 0.05 & -0.25 & 0.66 & 0.43 & 0.39 & 0.08 & 0.15 & 0.48 \\
\hline Loading & -0.37 & -0.19 & 0.05 & 0.37 & 0.11 & -0.04 & 0.53 & 0.27 & 0.56 & 0.28 & -0.29 & 0.0 \\
\hline Foot Flat & 0.4 & 0.07 & 0.17 & -0.16 & -0.05 & 0.12 & -0.63 & -0.15 & -0.6 & -0.22 & 0.04 & 0.06 \\
\hline Pushing & -0.32 & 0.24 & -0.22 & -0.18 & 0.01 & -0.29 & 0.5 & -0.21 & 0.45 & -0.16 & 0.15 & 0.17 \\
\hline Double Support & 0.46 & 0.25 & 0.17 & 0.17 & -0.15 & 0.11 & -0.75 & -0.53 & -0.49 & -0.2 & -0.02 & -0.26 \\
\hline Stride Length & -0.7 & -0.01 & 0.01 & 0.23 & 0.61 & 0.38 & 0.79 & 0.12 & 1.0 & 0.54 & -0.25 & -0.3 \\
\hline Peak Swing & -0.61 & -0.51 & -0.07 & -0.02 & 0.17 & 0.12 & 0.78 & 0.52 & 0.57 & 0.39 & -0.05 & -0.13 \\
\hline \multicolumn{13}{|l|}{ Foot Clearance Variables } \\
\hline Strike Angle & -0.63 & -0.39 & 0.08 & 0.41 & 0.36 & 0.0 & 0.62 & 0.25 & 0.73 & 0.19 & -0.29 & 0.07 \\
\hline Lift-Off $\mathrm{Al}$ & 0.7 & -0.04 & -0.22 & 0.22 & -0.43 & 0.17 & -0.6 & 0.04 & -0.77 & 0.25 & 0.26 & -0.34 \\
\hline Maximum Heel Clearance & -0.51 & -0.34 & 0.38 & 0.27 & 0.48 & -0.07 & 0.33 & 0.3 & 0.46 & 0.22 & -0.47 & 0.19 \\
\hline Maximum Toe Clearance 1 & -0.05 & 0.02 & 0.07 & 0.28 & 0.12 & -0.11 & 0.2 & 0.12 & 0.11 & -0.07 & -0.28 & 0.23 \\
\hline Minimum Toe Clearance & 0.6 & -0.16 & -0.29 & -0.21 & -0.28 & 0.11 & -0.31 & 0.26 & -0.45 & 0.13 & 0.04 & -0.01 \\
\hline Maximum Toe Clearance 2 & -0.67 & 0.05 & 0.13 & 0.02 & 0.55 & -0.25 & 0.53 & -0.09 & 0.69 & -0.19 & -0.34 & 0.4 \\
\hline
\end{tabular}

Raw Data

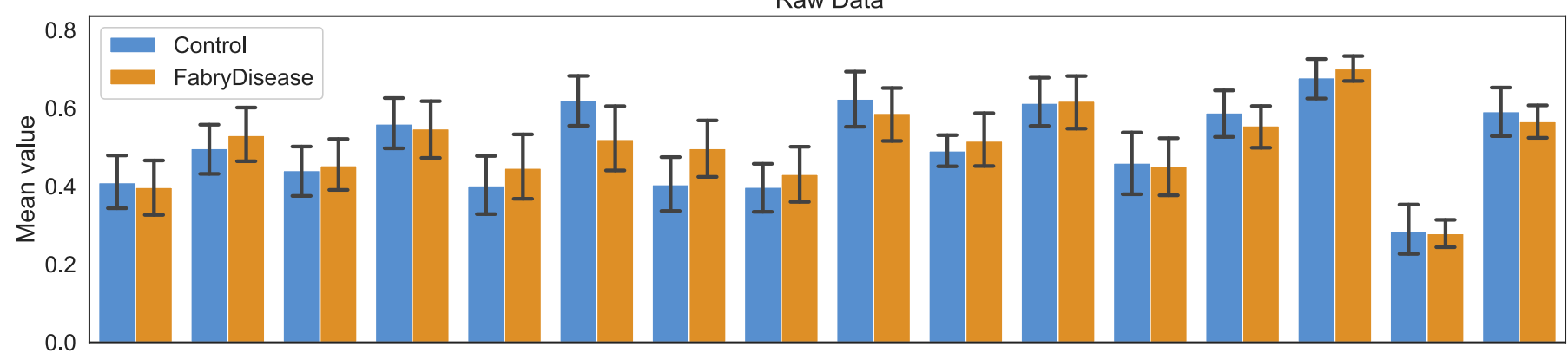

MR Normalized Data

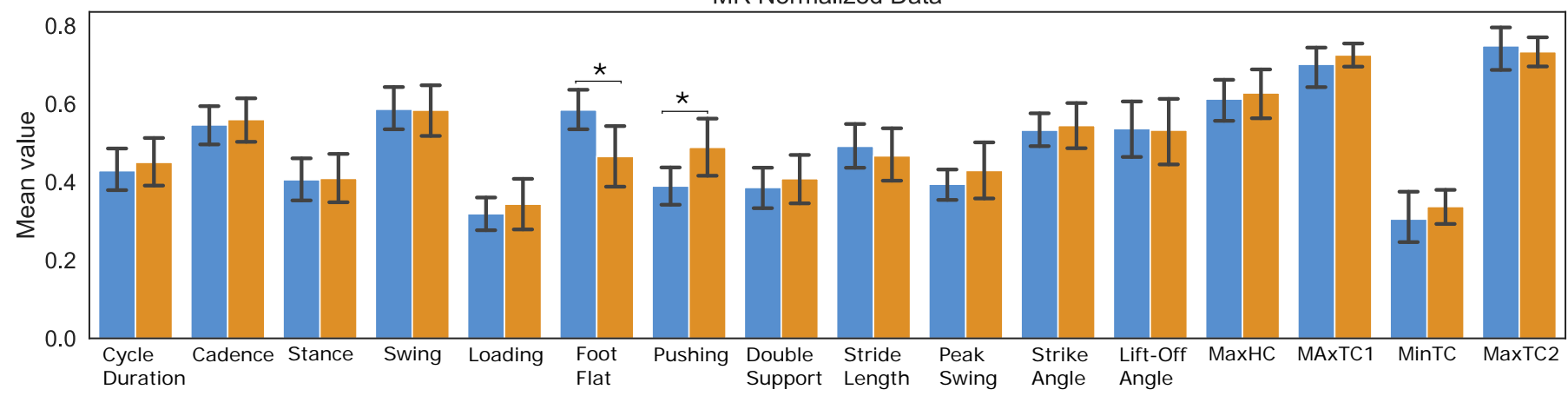

Fig. 1. Comparison between the mean value of gait features in patient with FD and controls. Data are shown for the MR normalized gait and the raw gait data. Significant differences in gait features between FD patients and controls are indicated with one asterisk $(* p<.05)$. Whiskers represent $95 \%$ confidence interval (CI) values. The data was scaled between 0 and 1 to fit onto the same plot.

formance increase when based on the MR normalized gait variables, the RF classifier accuracy increased from $59.5 \%$ when using raw gait data to $76.4 \%$ when using the MR normalized gait data, it seems that the RF classifier benefits the most from the MR normalization approach. These findings are also alined with a previous study where a RF classifier went from being the worst classifier when classifying PD gait patterns based on raw gait variables to the best classifier when based on MR normalized gait variables, the accuracy increased from $75.0 \%$ to $92.6 \%$ [34]. We also observed that the AUC measurements indicate significant improvements in the classifiers performance when based on the MR normalized gait data, the only exception was the DBN classifier which yielded the same AUC performance with raw or MR normalized gait data. A previous study indicates that the ROC curves and AUC measure should be used with caution unless one has a very large dataset, according to this study smaller datasets might lead to inaccurate results [18]. However, our results indicate 
TABLE VI

CLASSIFICATION PERFORMANCE MEASURES OBTAINED WITH EACH CLASSIFIER BASED ON THE PRINCIPAL COMPONENTS DERIVED FROM THE RAW AND MR NORMALIZED GAIT VARIABLES. VALUES IN PARENTHESES INDICATE STANDARD DEVIATION OF THE PERFORMANCE. ALL PERFORMANCE RESULTS ARE IN PERCENTAGE.

\begin{tabular}{|c|c|c|c|c|c|}
\hline \multirow{3}{*}{\multicolumn{2}{|c|}{ Classifier }} & \multicolumn{4}{|c|}{ Controls vs. FD patients } \\
\hline & & \multicolumn{2}{|c|}{ Raw Dataset } & \multicolumn{2}{|c|}{ MR Normalized Dataset } \\
\hline & & Mean & $95 \%$ CI & Mean & $95 \%$ CI \\
\hline \multirow{3}{*}{ MLP } & Accuracy & $68.87( \pm 16.08)$ & $(56.75: 80.99)$ & $72.20( \pm 15.11)$ & $(60.81: 83.59)$ \\
\hline & Sensitivity & $70.0( \pm 19.05)$ & $(55.62: 84.38)$ & $76.83( \pm 20.20)$ & $(61.60: 92.06)$ \\
\hline & Specificity & $71.33( \pm 19.5)$ & $(56.63: 86.04)$ & $72.33( \pm 16.33)$ & $(60.02: 84.65)$ \\
\hline \multirow{3}{*}{ DBN } & Accuracy & $61.55( \pm 11.27)$ & (53.05:70.04) & $65.12( \pm 13.87)$ & (54.66:75.57) \\
\hline & Specificity & $62.0( \pm 14.47)$ & $(51.09: 72.91)$ & $64.33( \pm 17.05)$ & $(51.48: 77.19)$ \\
\hline & Sensitivity & $64.83( \pm 15.75)$ & $(52.96: 76.71)$ & $67.33( \pm 14.95)$ & $(56.06: 78.60)$ \\
\hline \multirow{3}{*}{ SVM } & Accuracy & $71.96( \pm 12.57)$ & $(62.48: 81.44)$ & $78.21( \pm 9.63)$ & $(70.95: 85.47)$ \\
\hline & Sensitivity & $75.83( \pm 18.05)$ & $(62.22: 89.44)$ & $76.89( \pm 12.57)$ & $(67.35: 86.31)$ \\
\hline & Specificity & $73.0( \pm 16.41)$ & $(60.62: 85.38)$ & $88.50( \pm 14.67)$ & $(77.44: 99.56)$ \\
\hline \multirow{3}{*}{$\mathbf{R F}$} & Accuracy & $59.52( \pm 14.86)$ & $(48.32: 70.73)$ & $76.37( \pm 10.78)$ & $(68.24: 84.50)$ \\
\hline & Sensitivity & $58.50( \pm 24.16)$ & $(40.28: 76.72)$ & $80.50( \pm 18.23)$ & $(66.76: 94.24)$ \\
\hline & Specificity & $70.50( \pm 21.86)$ & $(54.02: 86.98)$ & $84.67( \pm 16.91)$ & $(72.14: 97.19)$ \\
\hline
\end{tabular}
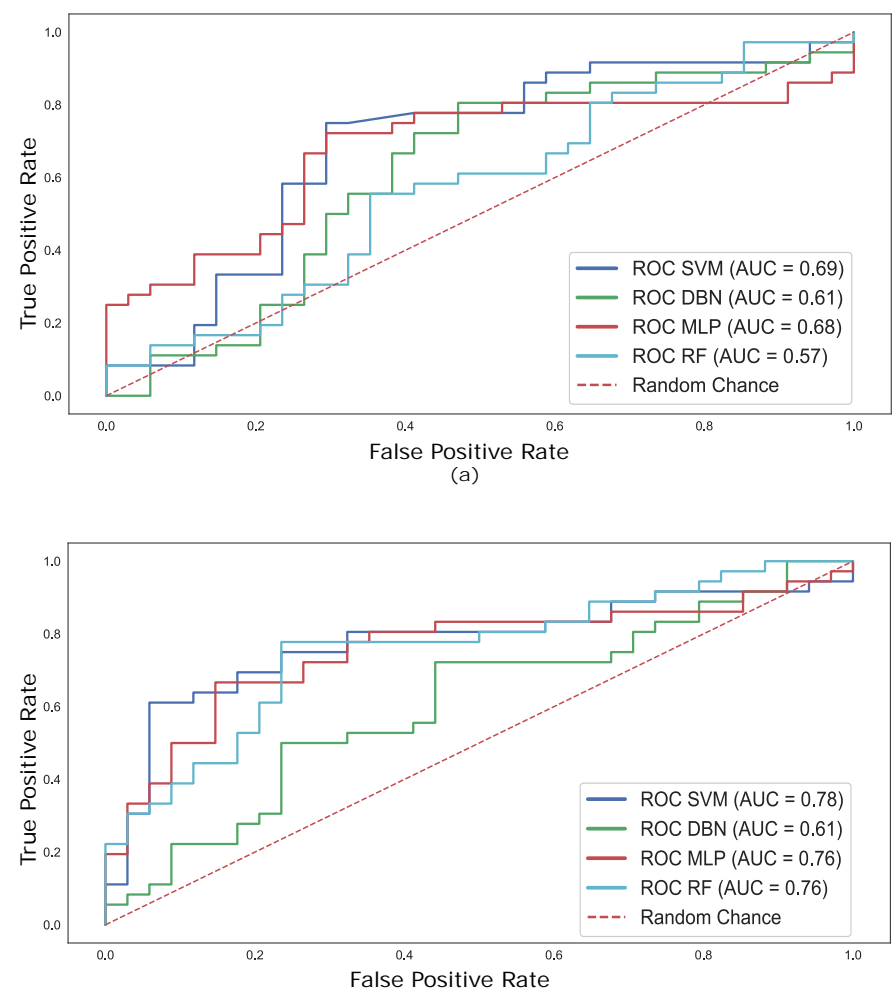

(b)

Fig. 2. ROC curves for the developed machine learning classifiers applied to (a) raw gait data, (b) MR normalized gait data. Performance is shown for the following machine learning classifiers: DBN, MLP, SVM and RF.

significant improvements over all the evaluated metrics (not only AUC and ROC curves) when the classifiers are based on MR normalized gait data.

There are various limitations that should be addressed to correctly interpret our results. Firstly, the developed MR models were based on a fairly small number of control subjects ( $n=34)$, some weak correlations were still present after normalization which may be related to the restricted number of controls or may also be related to the fact that non-linearities have been observed in gait variability, specially in people above 60 years old [10], [11], the developed MR models are not able to capture non-linearities. Nevertheless, a larger number of control subjects might improve MR models and further reduce the correlations. It is important to also keep in mind that there are other factors besides speed, stride length and physical properties that impact gait variables, an example is cognition [5]. For future research it would be interesting to collect a greater group of control subjects and collect cognitive measures to further evaluate the capabilities of MR normalization for the de-correlation of gait variables and ability to increase the classification performances.

Finally and most importantly, to the best of our knowledge, this is the first work that includes a FD group and analysis the capabilities of different machine learning classifiers for the prediction of FD when based on gait data. The results obtained in this work are extremely promising for the diagnosis and evaluation of FD.

\section{CONCLUSION}

Inter-subject variability in physical properties, speed and stride length, significantly impacts final gait performance. Our results show that spatial-temporal gait variables are predominantly affected by speed, whereas foot clearance gait variables seem to be predominantly affected by stride length. A MR normalization approach is able to de-correlate spatial-temporal and foot clearance gait variables from physical properties, speed and stride length.

In this work, all classifiers improved their performance when based on the MR normalized gait variables. The comparative classification using four different classifiers suggests that the superior classifier at every performance measure was the SVM, both when based on raw and MR normalized gait variables. Random Forest classifiers showed an extremely significant performance increase when based on the MR normalized gait achieving the second best overall performance.

Machine learning classifiers, specially SVMs and RFs, using gait variables normalized using a MR approach can reasonably 
support doctors in the objective of diagnosing and evaluating FD.

\section{REFERENCES}

[1] Martín Abadi, Ashish Agarwal, Paul Barham, Eugene Brevdo, Zhifeng Chen, Craig Citro, Greg S. Corrado, Andy Davis, Jeffrey Dean, Matthieu Devin, Sanjay Ghemawat, Ian Goodfellow, Andrew Harp, Geoffrey Irving, Michael Isard, Yangqing Jia, Rafal Jozefowicz, Lukasz Kaiser, Manjunath Kudlur, Josh Levenberg, Dan Mané, Rajat Monga, Sherry Moore, Derek Murray, Chris Olah, Mike Schuster, Jonathon Shlens, Benoit Steiner, Ilya Sutskever, Kunal Talwar, Paul Tucker, Vincent Vanhoucke, Vijay Vasudevan, Fernanda Viégas, Oriol Vinyals, Pete Warden, Martin Wattenberg, Martin Wicke, Yuan Yu, and Xiaoqiang Zheng. TensorFlow: Large-scale machine learning on heterogeneous systems, 2015. Software available from tensorflow.org.

[2] Hervé Abdi and Lynne J. Williams. Principal component analysis. Wiley Interdisciplinary Reviews: Computational Statistics, 2(4):433-459, jul 2010.

[3] Satyabrata Aich, Ki-Won Choi, Pyari Mohan Pradhan, Jinse Park, and Hee-Cheol Kim. A performance comparison based on machine learning approaches to distinguish parkinsons disease from alzheimer disease using spatiotemporal gait signals. Advanced Science Letters, 24(3):2058-2062, 2018.

[4] Lisa Alcock, Brook Galna, Ruth Perkins, Sue Lord, and Lynn Rochester. Step length determines minimum toe clearance in older adults and people with parkinsons disease. Journal of biomechanics, 71:30-36, 2018.

[5] Marianna Amboni, Paolo Barone, and Jeffrey M Hausdorff. Cognitive contributions to gait and falls: evidence and implications. Movement disorders, 28(11):1520-1533, 2013.

[6] Zoltán Bejek, Róbert Paróczai, Árpád Illyés, and Rita M Kiss. The influence of walking speed on gait parameters in healthy people and in patients with osteoarthritis. Knee Surgery, Sports Traumatology, Arthroscopy, 14(7):612-622, 2006.

[7] David A Belsley. A guide to using the collinearity diagnostics. Computer Science in Economics and Management, 4(1):33-50, 1991.

[8] Bernhard E. Boser, Bernhard E. Boser, Isabelle M. Guyon, and Vladimir N. Vapnik. A Training Algorithm for Optimal Margin Classifiers. PROCEEDINGS OF THE 5TH ANNUAL ACM WORKSHOP ON COMPUTATIONAL LEARNING THEORY, pages 144-152, 1992.

[9] Leo Breiman. Random forests. Machine Learning, 45(1):5-32, Oct 2001.

[10] Ugo H Buzzi, Nicholas Stergiou, Max J Kurz, Patricia A Hageman, and Jack Heidel. Nonlinear dynamics indicates aging affects variability during gait. Clinical biomechanics, 18(5):435-443, 2003.

[11] Michele L Callisaya, Leigh Blizzard, Michael D Schmidt, Jennifer L McGinley, and Velandai K Srikanth. Ageing and gait variabilitya population-based study of older people. Age and ageing, 39(2):191197, 2010.

[12] François Chollet et al. Keras. github, https://github.com/fchollet/keras, 2015.

[13] Carlos Fernandes, Luís Fonseca, Flora Ferreira, Miguel Gago, Lus Costa, Nuno Sousa, Carlos Ferreira, Joao Gama, Wolfram Erlhagen, and Estela Bicho. Artificial neural networks classification of patients with parkinsonism based on gait. In 2018 IEEE International Conference on Bioinformatics and Biomedicine (BIBM), pages 2024-2030. IEEE, 2018.

[14] Flora Ferreira, Miguel F Gago, Estela Bicho, Catarina Carvalho, Nafiseh Mollaei, Lurdes Rodrigues, Nuno Sousa, Pedro Pereira Rodrigues, Carlos Ferreira, and João Gama. Gait stride-to-stride variability and foot clearance pattern analysis in idiopathic parkinsons disease and vascular parkinsonism. Journal of biomechanics, 2019.

[15] Asja Fischer and Christian Igel. Training restricted Boltzmann machines: An introduction. Pattern Recognition, 47(1):25-39, jan 2014.

[16] Roberto Giugliani, Dau-Ming Niu, Uma Ramaswami, Michael West, Derralynn Hughes, Christoph Kampmann, Guillem Pintos-Morell, Kathleen Nicholls, Jörn-Magnus Schenk, and Michael Beck. A 15-year perspective of the fabry outcome survey. Journal of Inborn Errors of Metabolism and Screening, 4:2326409816666298, 2016.

[17] Jiawei Han, Micheline Kamber, and Jian Pei. Data Mining: Concepts and Techniques. Elsevier Inc., 2012.

[18] Blaise Hanczar, Jianping Hua, Chao Sima, John Weinstein, Michael Bittner, and Edward R Dougherty. Small-sample precision of roc-related estimates. Bioinformatics, 26(6):822-830, 2010.
[19] Simon S. Haykin and Simon S. Haykin. Neural networks and learning machines. Prentice Hall/Pearson, 2009.

[20] Geoffrey E. Hinton, Simon Osindero, and Yee Whye Teh. A fast learning algorithm for deep belief nets. Neural Computation, 18(7):1527-1554, 2006.

[21] Chris Kirtley, Michael W Whittle, and RJ Jefferson. Influence of walking speed on gait parameters. Journal of biomedical engineering, 7(4):282288, 1985.

[22] Edwin Kolodny, Andreas Fellgiebel, Max J Hilz, Katherine Sims, Pau Caruso, Thanh G Phan, Juan Politei, Renzo Manara, and Alessandro Burlina. Cerebrovascular involvement in fabry disease: current status of knowledge. Stroke, 46(1):302-313, 2015.

[23] Ken J Kubota, Jason A Chen, and Max A Little. Machine learning for large-scale wearable sensor data in parkinson's disease: Concepts, promises, pitfalls, and futures. Movement disorders, 31(9):1314-1326, 2016.

[24] Joseph Lev. The Point Biserial Coefficient of Correlation. The Annals of Mathematical Statistics, 20(1):125-126, mar 1949.

[25] Matthias Löhle, Derralynn Hughes, Alan Milligan, Linda Richfield, Heinz Reichmann, Atul Mehta, and Anthony HV Schapira. Clinical prodromes of neurodegeneration in anderson-fabry disease. Neurology, 84(14):1454-1464, 2015.

[26] Sue Lord, Brook Galna, and Lynn Rochester. Moving forward on gait measurement: toward a more refined approach. Movement Disorders, 28(11):1534-1543, 2013.

[27] Andrea Mannini, Diana Trojaniello, Andrea Cereatti, and Angelo Sabatini. A machine learning framework for gait classification using inertial sensors: Application to elderly, post-stroke and huntingtons disease patients. Sensors, 16(1):134, 2016.

[28] Val Mikos, Shih-Cheng Yen, Arthur Tay, Chun-Huat Heng, Chloe Lau Ha Chung, Sylvia Hui Xin Liew, Dawn May Leng Tan, and Wing Lok Au. Regression analysis of gait parameters and mobility measures in a healthy cohort for subject-specific normative values. PloS one, 13(6): $\mathrm{e} 0199215,2018$

[29] F. Pedregosa, G. Varoquaux, A. Gramfort, V. Michel, B. Thirion, O. Grisel, M. Blondel, P. Prettenhofer, R. Weiss, V. Dubourg, J. Vanderplas, A. Passos, D. Cournapeau, M. Brucher, M. Perrot, and E. Duchesnay. Scikit-learn: Machine learning in Python. Journal of Machine Learning Research, 12:2825-2830, 2011.

[30] Erika Rovini, Carlo Maremmani, Alessandra Moschetti, Dario Esposito, and Filippo Cavallo. Comparative motor pre-clinical assessment in parkinsons disease using supervised machine learning approaches. Annals of biomedical engineering, 46(12):2057-2068, 2018

[31] David G. Stork. Solution Manual to accompany Pattern Classification. page 680, 2000.

[32] Nooritawati Md Tahir and Hany Hazfiza Manap. Parkinson disease gait classification based on machine learning approach. J. Appl. Sci. Faisalabad (Faisalabad), 12:180-185, 2012

[33] Ferdous Wahid, Rezaul Begg, Noel Lythgo, Chris J Hass, Saman Halgamuge, and David C Ackland. A multiple regression approach to normalization of spatiotemporal gait features. Journal of applied biomechanics, 32(2):128-139, 2016.

[34] Ferdous Wahid, Rezaul K Begg, Chris J Hass, Saman Halgamuge, and David C Ackland. Classification of parkinson's disease gait using spatial-temporal gait features. IEEE journal of biomedical and health informatics, 19(6):1794-1802, 2015.

[35] Yi Xia, Qingwei Gao, and Qiang Ye. Classification of gait rhythm signals between patients with neuro-degenerative diseases and normal subjects: Experiments with statistical features and different classification models. Biomedical Signal Processing and Control, 18:254-262, 2015.

[36] Joseph A Zeni Jr and Jill S Higginson. Differences in gait parameters between healthy subjects and persons with moderate and severe knee osteoarthritis: a result of altered walking speed? Clinical biomechanics, 24(4):372-378, 2009 